\title{
Comparação entre dispositivos de galgamento costeiro com rampa única e com rampa dupla empregando espectro de ondas e design construtal
}

Comparison between coastal overtopping devices with single and double ramp using a wave spectrum and constructal design

\author{
J. C. Martins ${ }^{1 *}$; M. M. Goulart ${ }^{2}$; E. D. dos Santos²; L. A. Isoldi' ; M. N. Gomes ${ }^{3}$; \\ L. A. O. Rocha ${ }^{4}$ \\ ${ }^{1}$ Escola de Engenharia/PROMEC, Universidade Federal do Rio Grande do Sul, 90050-170, Porto Alegre-RS, Brasil \\ ${ }^{2}$ Centro de Ciências Computacionais, Escola De Engenharia e Instituto De Matemática, Estatística E Física/PPGMC, \\ Universidade Federal do Rio Grande, 96203-900, Rio Grande-RS, Brasil \\ ${ }^{3}$ Instituto Federal do Paraná/Campus Paranaguá/PPGCTS, Instituto Federal de Educação, Ciência e Tecnologia do \\ Estado do Paraná, 83215-750, Paranaguá-PR, Brasil
}

${ }^{4}$ Escola Politécnica/PPGEM, Universidade do Vale do Rio dos Sinos, 93022-750, São Leopoldo-RS, Brasil

*jaifercm@gmail.com

\begin{abstract}
Estudos sobre formas alternativas de geração de energia elétrica vêm aumentado nos últimos anos. Os conversores de energia das ondas (wave energy converter - WEC) são uma destas formas, dentre elas o dispositivo de galgamento destaca-se por seu princípio de funcionamento. No presente trabalho é avaliado geometricamente um dispositivo de galgamento com rampa dupla, com a finalidade de maximizar a potência disponível $\left(\left(P_{\mathrm{d}}\right)_{\mathrm{m}}\right)$, este resultado é então comparado com o obtido para um dispositivo com rampa única, este referente a um trabalho encontrado na literatura. O método Design Construtal é usado na avaliação geométrica (determinação de um campo de busca) e o método Busca Exaustiva para a otimização. Para a geração da onda se utilizou o espectro de ondas Pierson-Moskowitz (PM), com período de onda e altura significativa de, respectivamente, $T_{\mathrm{S}}=7,5 \mathrm{~s}$ e $H_{\mathrm{S}}=1,5 \mathrm{~m}$. As dimensões das rampas do dispositivo são obtidas em relação a fração de área da rampa $\left(\phi_{1}=0,0006 ; 0,0009 ; 0,0012\right.$ e 0,$0015 ;$ e $\phi_{2}=$ 0,0006 , além das razões entre as alturas e comprimentos das rampas iguais: $H_{1} / L_{1}=0,3$ e $H_{2} / L_{2}=0,2$ e 0,3 . Os resultados mostram que, para o dispositivo com rampa dupla, a aplicação do Design Construtal foi importante para avaliação geométrica, possibilitando determinar a potência disponível máxima para as condições impostas. Porém, em comparação com o dispositivo com rampa única, este mostrou-se uma opção melhor.

Palavras-chave: energia renovável, galgamento, ondas do mar.
\end{abstract}

Studies about alternatives of generation of electricity have been increasing in last years. Wave energy converters (WEC) are one of these forms, among them the overtopping device stands out by your operating principle. In the present work a overtopping device with a double ramp is evaluated geometrically in order to maximize the available power $\left(\left(P_{\mathrm{d}}\right)_{\mathrm{m}}\right)$, this result are compared to a work found in literature about one device with a single ramp. The Constructal Design method is used in the geometric evaluation (determination of a search field) and the Exhaustive Search method for optimization. For the wave generation, the Pierson-Moskowitz (PM) wave spectrum was used, with a wave period and the significant height of, respectively, $T_{\mathrm{S}}=7,5 \mathrm{~s}$ and $H_{\mathrm{S}}=1,5 \mathrm{~m}$. The ramp dimensions of the device are obtained to the fraction of ramps area $\left(\phi_{1}=0,0006 ; 0,0009 ; 0,0012\right.$ and 0,0015 ; and $\left.\phi_{2}=0,0006\right)$, besides the ratios between heights and lengths of ramps $H_{1} / L_{1}=0,3$ e $H_{2} / L_{2}=0,2$ e 0,3 . The results show that, for the double ramp device, the application of the Constructal Design was important for geometric evaluation, making it possible to determine the maximum available power for the conditions imposed. However, compared to the single ramp device, this proved to be a better option.

Keywords: renewable energy, overtopping, ocean waves. 


\section{INTRODUÇÃO}

Os conversores de energia das ondas (wave energy converter - WEC) são equipamentos desenvolvidos para produzir energia elétrica a partir das ondas do mar. Estes são uma das formas para tentar diminuir o problema energético mundial atual e futuro. Por exemplo, estima-se que na costa do sul do Brasil um potencial de aproximadamente $30 \mathrm{~kW}$ por metro de onda [1]. Diversos equipamentos vêm sendo estudados nos últimos anos, entre eles o dispositivo de galgamento é um deles. Trata-se de um equipamento com uma rampa, sobre a qual a água do mar incidente passa sobre ela, acumulando-se em um reservatório, conforme Figura 1. A água então escoa por uma turbina de baixa queda retornando ao mar [2].

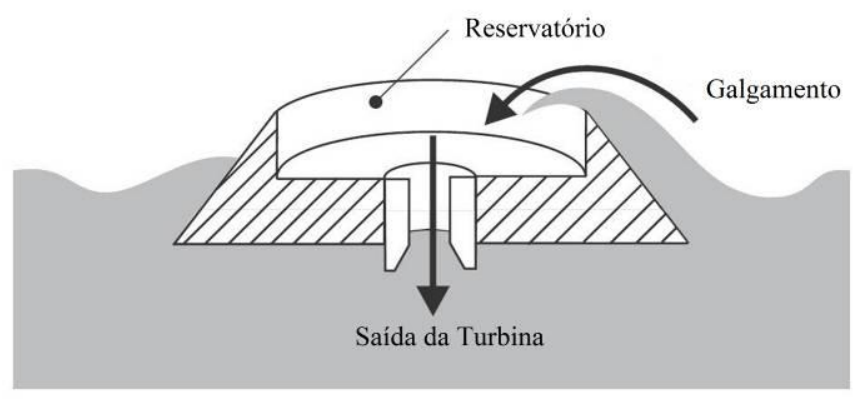

Figura 1: Ilustração do princípio de funcionamento do dispositivo de galgamento.

Alguns dos estudos sobre o dispositivo de galgamento realizados mais recentes abordaram sobre a avaliação experimental de parâmetros do dispositivos $[3,4,5,6]$; trabalhos numéricos [7, $8,9,10]$ e com aplicação do método Design Construtal para avaliação geométrica $[11,12,13,14$, 15].

Portanto, o objetivo do trabalho é realizar uma avaliação geométrica aplicando o método Design Construtal $[16,17]$ juntamente com o método Busca Exaustiva (para otimização) de um dispositivo de galgamento com rampa dupla em escala real e comparar a evolução da função objetivo (potência disponível $\left(P_{\mathrm{d}}\right)$ ) em função das restrições (área total do canal e área da rampa) e dos graus de liberdade (altura e comprimento da rampa superior; fração de área das rampas e distância entre as rampas) com um dispositivo de rampa única estudado em Martins et al. (2017) [14]. Para isso, usaram-se quatro valores para a fração de área da rampa $\left(\phi_{1}\right): 0,0006 ; 0,0009$; 0,0012 e 0,0015; e um valor para $\phi_{2}: 0,0006$. Além disso, uma razão entre altura e comprimento da rampa inferior $\left(H_{1} / L_{1}\right): 0,3$; e dois valores para razão da rampa superior $\left(H_{2} / L_{2}\right): 0,2$ e 0,3 . O pré-processamento foi realizado com o software Ansys Workbench, o processamento com o Ansys Fluent v.16 (2015) [18] e o pós-processamento com o auxílio de uma planilha eletrônica.

\section{MODELAGEM MATEMÁTICA E NUMÉRICA}

A Figura 2 mostra o domínio estudado, um dispositivo de galgamento bidimensional ( $x$ na direção horizontal e $z$ na direção vertical) com duas rampas, colocado em um canal de ondas em escala real, onde a terceira dimensão $(y)$ fica perpendicular ao plano da figura.

O desenvolvimento do espectro de onda (Pierson-Moskowitz) deu-se pela imposição de um campo de velocidades na metade inferior da superfície esquerda do canal (linha azul - velocidade prescrita) com a geração de 15 componentes de onda. As características de onda empregadas foram: altura significativa $\left(H_{s}\right)$ igual a $1,5 \mathrm{~m}$ e período $(T)$ de $7,5 \mathrm{~s}$ (comprimento de onda $\left(\lambda_{\mathrm{s}}\right)$ de $65,4 \mathrm{~m})$. A costa do Rio Grande do Sul apresenta ondas de média a elevada energia, com altura significativa $\left(H_{s}\right)$ de $1,5 \mathrm{~m}$ e período $(T)$ entre 7,0 a 9,0 s [19], características estas avaliadas numericamente por Lisboa et al. (2016) [20].

A profundidade da superfície livre da água $(h)$ foi de $10,0 \mathrm{~m}$. Já o canal foi desenhado com uma altura $\left(H_{\mathrm{T}}\right)$ igual a $20,0 \mathrm{~m}\left(\sim 13,33 * H_{s}\right)$ e comprimento $\left(L_{\mathrm{T}}\right)$ de $327,0 \mathrm{~m}\left(5 * \lambda_{\mathrm{s}}\right)$, ficando com uma razão $H_{\mathrm{T}} / L_{\mathrm{T}}$ de 0,0612 . 
Com relação as dimensões do dispositivo de galgamento, tem-se: comprimentos das rampas $L_{1}$ e $L_{2}$; alturas das rampas $H_{1}$ e $H_{2}$; áreas das rampas $A_{\mathrm{r}, 1}$ e $A_{\mathrm{r}, 2}$; comprimentos dos reservatórios $L_{\mathrm{R}, 1}$ (igual a $20 \mathrm{~m}$ ) e $L_{\mathrm{R}, 2}$ (igual a $13,885 \mathrm{~m}$ ) e alturas $H_{\mathrm{R}, 1}$ e $H_{\mathrm{R}, 2}$, além das alturas $h_{\mathrm{a}, 1}$ e $h_{\mathrm{a}, 2}$ representando a altura de água acumulada nos reservatórios, e uma distância entre as rampas $L_{\mathrm{g}}$ $(1,0 \mathrm{~m})$. As frações de área da rampa inferior $\left(\phi_{1}\right)$ foram: 0,$0006 ; 0,0009 ; 0,0012$ e 0,0015 ; e para $\phi_{2}$ somente o valor 0,0006 . E a razão entre a altura e comprimento da rampa $H_{1} / L_{1}$ o valor utilizado foi de $0,3\left(\sim 16,7^{\circ}\right)$, e para $H_{2} / L_{2}$ dois valores: $0,2\left(\sim 11,3^{\circ}\right)$ e 0,3 .

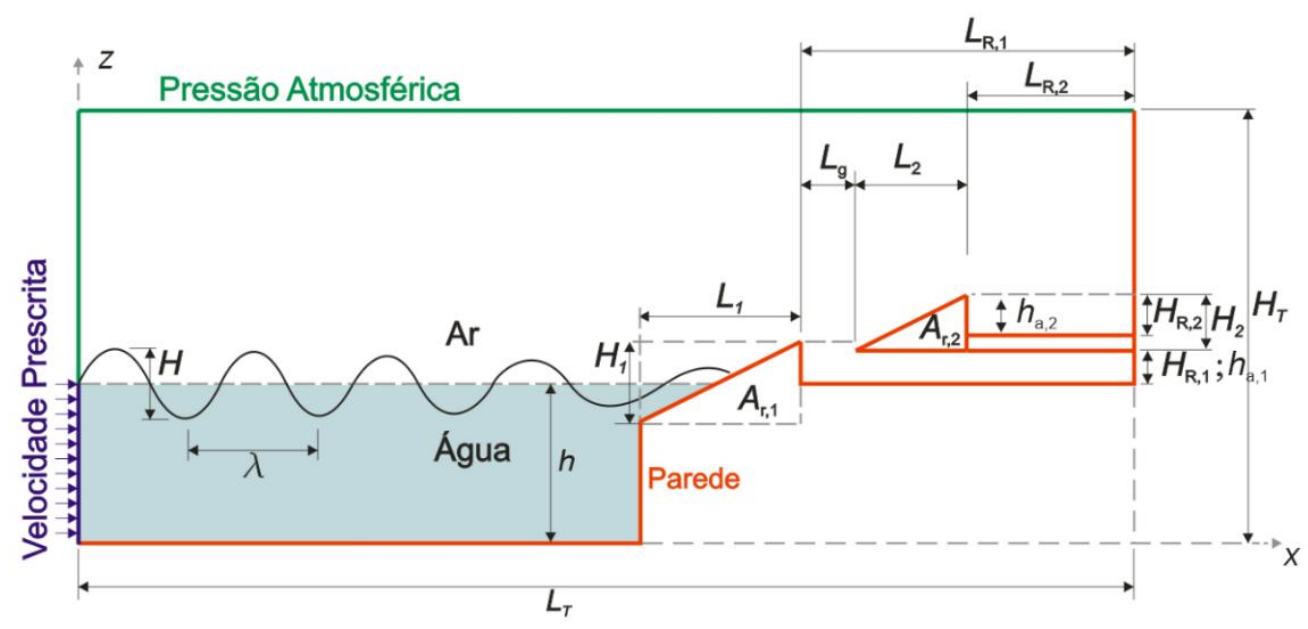

Figura 2: Domínio computacional do dispositivo de galgamento analisado.

Dessa maneira é possível avaliar a influência das frações $\phi_{1}$ e $\phi_{2}$, e das razões $H_{1} / L_{1}$ e $H_{2} / L_{2}$ sobre a potência disponível $\left(P_{\mathrm{d}}\right)$, e comparar o resultado com aqueles obtidos por Martins et al. (2017)[14] para um dispositivo de galgamento com rampa única.

\subsection{DESIGN CONSTRUTAL APLICADO AO DISPOSITIVO DE GALGAMENTO}

O método Design Construtal é utilizado no presente estudo com a finalidade de determinar o campo de busca das geometrias do dispositivo de galgamento, e para a otimização o método de Busca Exaustiva é utilizado. Desta forma é possível reconhecer quais são as geometrias ótimas (aquelas que conduzem a maximização da função objetivo), levando em consideração as condições físicas utilizadas.

Como restrições (duas), temos: a área total do canal (Eq. (1)), a área da rampa (Eq. (2)):

$$
\begin{aligned}
& A_{\mathrm{T}}=H_{\mathrm{T}} L_{\mathrm{T}} \\
& A_{\mathrm{r}, \mathrm{i}}=\frac{H_{\mathrm{i}} L_{\mathrm{i}}}{2}
\end{aligned}
$$

onde $\mathrm{i}=1$ e $2 ; H_{\mathrm{T}} \sim 13,33 * H_{\mathrm{s}}$ e $L_{\mathrm{T}}=5^{*} \lambda_{\mathrm{s}}$.

Define-se como fração de área das rampas, a equação:

$$
\phi_{\mathrm{i}}=\frac{A_{\mathrm{r}, \mathrm{i}}}{A_{\mathrm{T}}}
$$

Busca-se maximizar a potência disponível $\left(P_{\mathrm{d}}\right)$ por metro de frente de onda, para isso propõese a seguinte equação [15]:

$$
P_{\mathrm{d}}=\frac{g h_{\mathrm{a}, \mathrm{i}, \mathrm{t}_{\mathrm{f}}}}{t_{\mathrm{f}}} \int_{t_{\mathrm{i}}}^{t_{\mathrm{f}}} \dot{m}_{\mathrm{i}} d t
$$


onde $g$ é a aceleração da gravidade $\left(\mathrm{m} / \mathrm{s}^{2}\right), h_{\mathrm{a}, \mathrm{i}, t_{\mathrm{f}}}$ é a altura de água acumulada no interior de cada um dos reservatórios (m), $t_{\mathrm{i}}$ é o instante de tempo inicial (s), $t_{\mathrm{f}}$ é o instante de tempo final (s) neste caso, $t_{\mathrm{f}}=100 \mathrm{~s}-\mathrm{e} \dot{m}_{\mathrm{i}}$ é a vazão mássica da água acumulada em cada reservatório $(\mathrm{kg} / \mathrm{s})$.

A maneira como aplicou-se o Design Construtal na avaliação geométrica é apresentada na Figura 3. Desta forma, o processo deu-se da seguinte forma: com o valor de 0,3 para $H_{1} / L_{1}$; 0,0006 para $\phi_{1} ; 0,2$ para $H_{2} / L_{2}$ e 0,0006 para $\phi_{2}$; temos a primeira configuração simulada para avaliação. Assim, este procedimento vai sendo repetido para os demais valores, resultando num total de oito simulações.

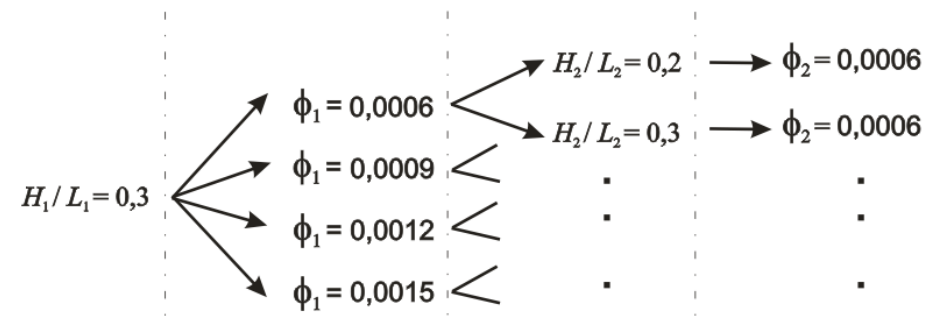

Figura 3: Ilustração do processo de avaliação geométrica do Design Construtal.

\subsection{O MODELO MULTIFÁSICO VOLUME OF FLUID (VOF)}

A avaliação da interação entre a água e o dispositivo, e da água com o ar, deu-se pelo o método Volume Of Fluid (VOF) (1981)[21], método multifásico o qual permite estudar escoamentos com duas ou mais fases, neste trabalho as duas fases (ar e água) são imiscíveis.

As equações de conservação de massa (Eq. (5)) e quantidade de movimento (Eq. (6)) para a mistura ar e água em um escoamento isotérmico, laminar e incompressível [22], são:

$$
\begin{gathered}
\frac{\partial \rho}{\partial t}+\nabla \cdot(\rho \vec{v})=0 \\
\frac{\partial \rho}{\partial t}(\rho \vec{v})+\nabla \cdot(\rho \vec{v} \vec{v})=-\nabla p+\nabla \cdot(\mu \overline{\bar{\tau}})+\rho \vec{g}
\end{gathered}
$$

onde $\rho$ é a massa específica da mistura $\left(\mathrm{kg} / \mathrm{m}^{3}\right) ; \vec{v}$ é o vetor velocidade do escoamento $(\mathrm{m} / \mathrm{s}) ; p$ é a pressão $\left(\mathrm{N} / \mathrm{m}^{2}\right) ; \rho \vec{g}$ são as forças de campo $\left(\mathrm{N} / \mathrm{m}^{3}\right)$ e $\overline{\bar{\tau}}$ é o tensor taxa de deformação $\left(\mathrm{N} / \mathrm{m}^{2}\right)$.

As propriedades do ar e da água empregadas foram: $\rho_{\mathrm{ar}}=1,225 \mathrm{~kg} / \mathrm{m}^{3} ; \rho_{\text {agua }}=998,2 \mathrm{~kg} / \mathrm{m}^{3} ; \mu_{\mathrm{ar}}$ $=1,789 \times 10^{-5} \mathrm{~kg} /(\mathrm{ms}) ; \mu_{\text {água }}=1,003 \times 10^{-3} \mathrm{~kg} /(\mathrm{ms})$.

Além disso, o conceito de fração de volume $\left(\alpha_{\mathrm{q}}\right)$ é usado por se tratar de um estudo com duas fases (ar e água) dentro de um volume de controle. Portanto, a soma das frações de volume deve ser unitária $\left(0 \leq \alpha_{\mathrm{q}} \leq 1\right)$. Assim, quando $\alpha_{\text {água }}=0$, o volume de controle está vazio de água e cheio de ar $\left(\alpha_{\mathrm{ar}}=1\right)$, já quando há uma mistura de ar e água, uma fase é o complemento da outra, ou seja, $\alpha_{\mathrm{ar}}=1-\alpha_{\text {agua. }}$ Logo, uma equação de transporte adicional para uma das frações de volume é necessária [21]:

$$
\frac{\partial(\alpha)}{\partial t}+\nabla \cdot(\alpha \vec{v})=0
$$

Já os valores de massa específica e viscosidade para a mistura, visto que as equações de conservação de massa e quantidade de movimento são resolvidas para a mistura, são:

$$
\begin{aligned}
& \rho=\alpha_{\text {água }} \rho_{\text {água }}+\alpha_{\mathrm{ar}} \rho_{\mathrm{ar}} \\
& \mu=\alpha_{\text {água }} \rho_{\text {água }}+\alpha_{\mathrm{ar}} \rho_{\mathrm{ar}}
\end{aligned}
$$




\subsection{ESPECTRO DE ONDAS - PIERSON-MOSKOWITZ (PM)}

O espectro de Pierson-Moskowitz foi empregado no presente estudo, sendo a densidade espectral, forma pela qual melhor pode-se comparar o espectro numérico com o analítico, dada por Chakrabarti (2005) [23]:

$$
S_{P M}(\omega)=\frac{5}{16} \frac{H_{S} \omega_{P}^{4}}{\omega^{5}} e^{-\left(\frac{5 \omega_{P}^{4}}{\omega^{4}}\right)}
$$

onde $H_{S}$ é a altura significativa da onda (m); $\omega_{\mathrm{P}}$ frequência de pico da onda (rad/s) e $\omega$ frequência da onda $(\mathrm{rad} / \mathrm{s})$.

As equações das componentes de velocidade horizontal $(u)$ e vertical $(w)$ são [23, 24, 25]:

$$
\begin{aligned}
& u=\zeta_{a_{n}} g k_{n} \frac{\cosh \left(k_{n} z+k_{n} h\right)}{\omega_{n} \cosh \left(k_{n} h\right)} \cos \left(k_{n} x-\omega_{n} t\right) \\
& w=\zeta_{a_{n}} g k_{n} \frac{\operatorname{senh}\left(k_{n} z+k_{n} h\right)}{\omega_{n} \operatorname{senh}\left(k_{n} h\right)} \operatorname{sen}\left(k_{n} x-\omega_{n} t\right)
\end{aligned}
$$

onde $\zeta_{a_{n}}$ é amplitude de uma componente (das 15 ondas) do espectro (m); $g$ aceleração da gravidade $\left(\mathrm{m} / \mathrm{s}^{2}\right) ; k_{n}$ número de onda $(\mathrm{rad} / \mathrm{m}) ; \omega_{n}$ frequência de uma componente $(\mathrm{rad} / \mathrm{s}) ; z$ posição entre a superfície livre e o fundo do canal (m); $x$ posição horizontal (m) e $t$ tempo (s).

\subsection{CONDIÇÕES DE CONTORNO}

O espectro de onda numérico é gerado no lado esquerdo inferior (linha azul - velocidade prescrita - Figura 2) do canal de ondas, sendo as componentes de velocidade nas direções horizontal $(x)$ e vertical $(z)$ dadas pelas equações (11) e (12).

$\mathrm{Na}$ região superior esquerda, Fig. 2, e na superfície superior (linha verde) se considerou pressão atmosférica $P_{\text {abs }}=101,3 \mathrm{kPa}$. Nas superfícies inferior, lateral direita, bem como, na superfície do dispositivo de galgamento (linha vermelha) impôs-se uma condição de impermeabilidade e não-deslizamento com velocidade nula $(u=w=0 \mathrm{~m} / \mathrm{s})$.

Como condições iniciais, considerou-se o fluido em movimento e a profundidade da água $(h)$ é igual a $10,0 \mathrm{~m}$.

\subsection{PROCEDIMENTOS NUMÉRICOS}

O estudo foi realizado com os softwares comerciais: Ansys Workbench 16 para o préprocessamento, criação da geometria e da malha; Ansys Fluent v.16 para o processamento, cálculo das equações diferenciais parciais (EDP); e o Microsoft Excel 2010 para o pósprocessamento. Utilizou-se um computador com processador AMD Quad-Core de 3,3 Ghz e 16 GB de memória RAM, empregando o Message Passing Interface (MPI) para a paralelização. O tempo de processamento foi de aproximadamente, $5,4 \times 10^{4} \mathrm{~s}(15 \mathrm{~h})$, para cada simulação.

O software Fluent v.16 (o qual utiliza o Método dos Volumes Finitos (MVF) [26]) foi usado para discretização das EDP's, e alguns dos parâmetros definidos foram: solver baseado na pressão; esquema de advecção Upwind de primeira ordem; Pressure Staggering Option (PRESTO) para a discretização espacial; o método Pressure-Implicit with Splitting of Operators (PISO) [27] para o acoplamento pressão-velocidade; método Geo-Reconstruction para a superfície ocupada pela água; fatores de sub-relaxação de 0,3 e 0,7 para as equações de conservação de massa e quantidade de movimento; consideraram-se convergidas as soluções quando os resíduos das equações de conservação de massa e quantidade de movimento nas direções horizontal e vertical ficaram menores do que $10^{-6}$.

A Figura 4 mostra o detalhe de refinamento da malha, na qual se empregou o método de malha Stretched com maior refinamento em regiões específicas [28]. Portanto, o número de volumes 
finitos retangulares das malhas ficou próximo de 180.000. Para a criação da malha adotou-se a recomendação teórica proposta por Gomes et al. (2012)[29] sobre o número adequado de volumes finitos para cada região do domínio.

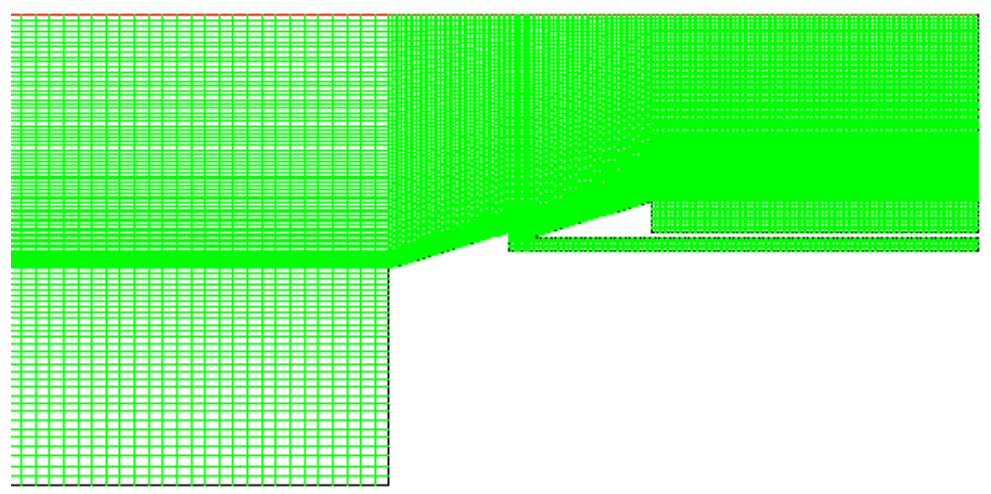

Figura 4: Ilustração da malha utilizada.

\section{RESULTADOS E DISCUSSÃO}

A comparação da elevação da superfície livre $(\eta)$ obtida de forma analítica e numérica para o espectro Pierson-Moskowitz, é apresentada na Figura 5a. Empregou-se a norma $l_{\infty}$ para comparar as alturas significativas [30]:

$$
\|x\|_{\infty}=\operatorname{máx}\left|x_{\mathrm{j}}\right|
$$

Logo, o valor obtido para a solução analítica da elevação da superfície foi igual a 1,2078 m e para a solução numérica $0,9732 \mathrm{~m}$, chegando a uma diferença entre os picos de $0,2346 \mathrm{~m}$.

Entretanto, para uma melhor avaliação do espectro de ondas, a análise mais adequada é em relação a densidade espectral, Figura 5b. Assim, com a aplicação da norma $l_{\infty}$ chegou-se nos seguintes valores: analítico, $0,2237 \mathrm{~m}^{2} \mathrm{~s}$ e numérico, $0,2452 \mathrm{~m}^{2} \mathrm{~s}$, ou seja, a uma diferença de $0,0215 \mathrm{~m}^{2} \mathrm{~s}$, portanto, pode-se considerar como uma reprodução próxima ao fenômeno real, conforme apresentado em Gomes et al. (2016)[31].
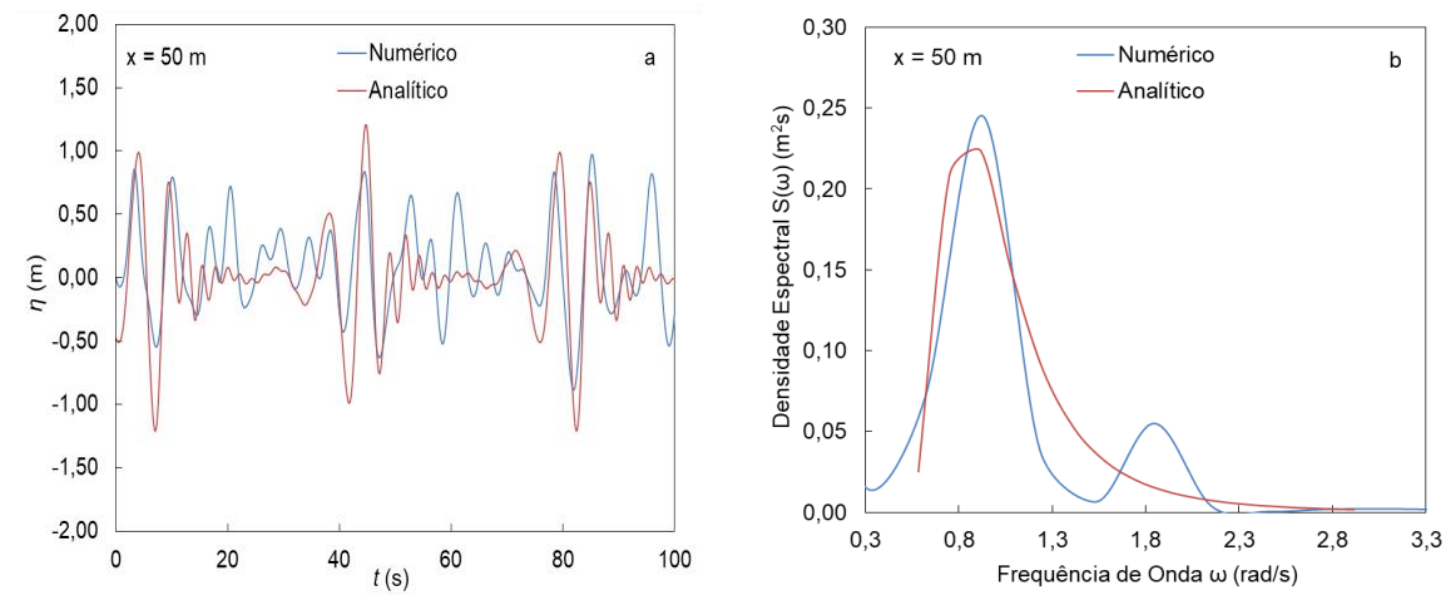

Figura 5: Comparação entre as soluções analítica e numérica: a) elevação da superfície livre; b) densidade espectral na posição $x=50 \mathrm{~m}$.

Conforme pode ser visto na Figura 6a, a maior potência disponível $\left(P_{\mathrm{d}}\right)$ foi obtida nas rampas inferiores, tanto para $H_{2} / L_{2}=0,2\left(\sim 11,3^{\circ}\right)$ quanto para $H_{2} / L_{2}=0,3\left(\sim 16,7^{\circ}\right)$, ou seja, para as duas razões entre altura e comprimento da rampa superior. Ainda, entre estas, o melhor caso deu-se 
quando $H_{1} / L_{1}=H_{2} / L_{2}=0,3$. Já para as rampas superiores a $P_{\mathrm{d}}$ obtida foi baixa, tendo como máxima o valor de, aproximadamente, $30 \mathrm{~W} / \mathrm{m}$, para razões entre altura e comprimento diferentes entre as duas rampas e fração de área da rampa inferior $\left(\phi_{1}\right)$ igual a 0,0015. E a Figura 6b, apresenta o resultado da soma das potências disponíveis da rampa inferior com a superior, para os dois casos, quando $H_{1} / L_{1}=H_{2} / L_{2}=0,3$ e para $H_{1} / L_{1}=0,3$ e $H_{2} / L_{2}=0,2$.
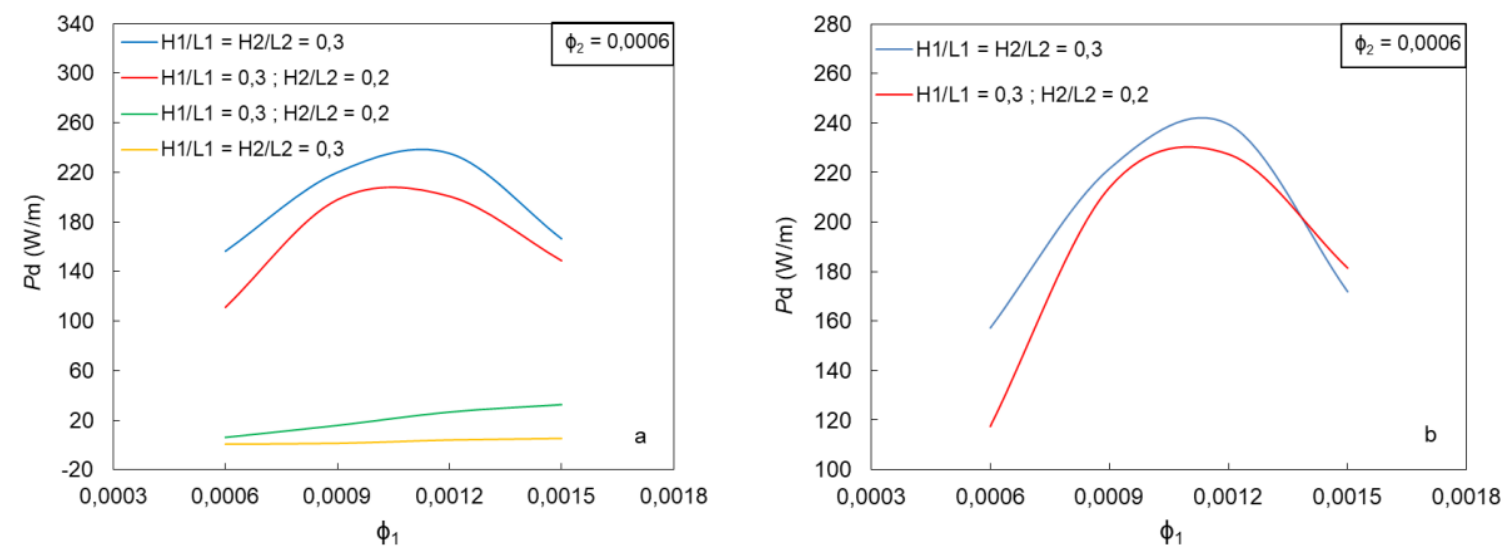

Figura 6: Comparação da potência disponível $\left(P_{d}\right)$ : a) com valores individuais da rampa inferior e rampa superior; b) em relação à soma dos valores das rampas inferior e superior.

Logo, os resultados mostraram como caso ótimo, para as condições físicas empregadas no estudo, a geometria de razão entre altura e comprimento das rampas iguais, ou seja, $H_{1} / L_{1}=H_{2} / L_{2}$ $=0,3$ e fração de área da rampa inferior $\phi_{1}=0,0012$, com a potência disponível máxima $\left(P_{\mathrm{d}}\right)_{\mathrm{m}}$ de $\sim 240 \mathrm{~W} / \mathrm{m}$.

Na Figura 7 apresenta-se a comparação entre os resultados obtidos no presente trabalho com os obtidos em Martins et al. (2017)[14] para um dispositivo com rampa única com uma razão $H_{1} / L_{1}$ $=0,3$ e fração de área da rampa $\phi=0,0006 ; 0,0009 ; 0,0012$ e 0,0015, com as mesmas condições de onda e canal.

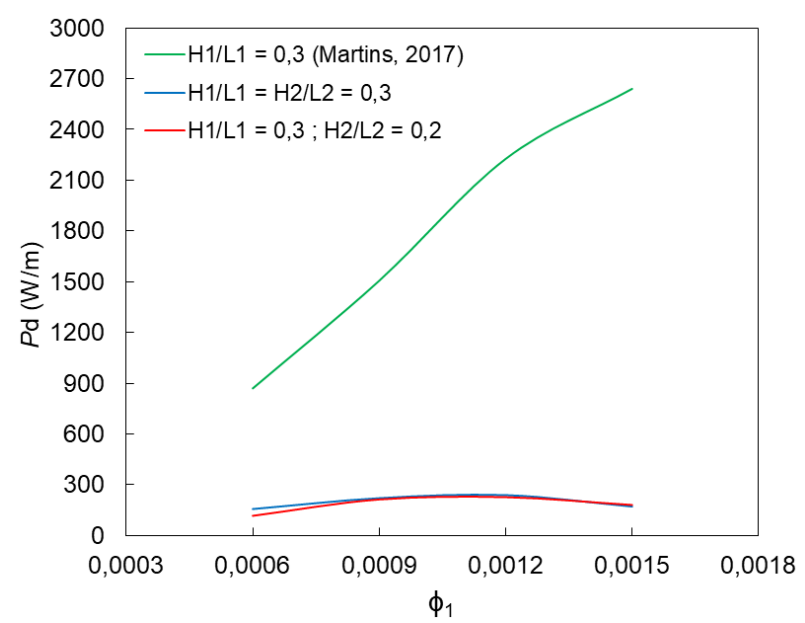

Figura 7: Comparação da potência disponível $\left(P_{d}\right)$ entre o dispositivo de galgamento com uma rampa (Martins, 2017[4]) e o com duas rampas.

Nota-se uma grande diferença entre a potência disponível obtida em cada um dos estudos, chegando a 11,25 vezes o valores da $P_{\mathrm{d}}$ entre elas, ou seja, $\left(P_{\mathrm{d}}\right)_{\mathrm{m}} \sim 240 \mathrm{~W} / \mathrm{m}$ (para o presente trabalho), $H_{1} / L_{1}=H_{2} / L_{2}=0,3, \phi_{1}=0,0012$ e $\phi_{2}=0,0006 ; \mathrm{e}\left(P_{\mathrm{d}}\right)_{\mathrm{m}} \sim 2700 \mathrm{~W} / \mathrm{m}$ (para [14]), $H_{1} / L_{1}=$ $0,3, \phi_{1}=0,0015$. Conclui-se que a segunda rampa restringiu a entra de água no reservatório gerando, assim, uma menor potência disponível. Portanto, nas condições de mar estudado, a melhor opção seria um dispositivo de galgamento de rampa única. Porém, a aplicação do Design Construtal considerando outras configurações de rampa são necessários para uma melhor avaliação em relação a maximização da potência do dispositivo. 


\section{CONCLUSÃO}

O presente estudo deu-se de modo numérico em busca da melhor geométrica de um dispositivo de galgamento costeiro em escala real com duas rampas, avaliando quatro valores para a fração de área da rampa inferior $\left(\phi_{1}\right)$ mantendo fixa a fração $\phi_{2}$, considerando ainda uma razão entre altura e comprimento da rampa inferior $\left(H_{1} / L_{1}\right)$ e duas razões para a rampa superior $\left(H_{2} / L_{2}\right)$. $\mathrm{O}$ Design Construtal foi empregado na determinação do campo de busca juntamente com o método de otimização Busca Exaustiva. Para o desenvolvimento da onda utilizou-se o espectro PiersonMoskowitz.

Em relação à avaliação geométrica, o método Design Construtal mostrou-se importante para desenvolvimento do estudo, e para o estabelecendo de recomendações teóricas para problemas de engenharia em situações e condições específicas.

Portanto, com os resultados foi possível determinar a potência disponível máxima $\left(P_{\mathrm{d}}\right)_{\mathrm{m}}$ para o dispositivo de rampa dupla, o valor encontrado foi de aproximadamente $240 \mathrm{~W} / \mathrm{m}$, para a configuração de rampas: $\phi_{1}=0,0012 ; \phi_{2}=0,0006 ; H_{1} / L_{1}=H_{1} / L_{1}=0,3\left(\sim 16,7^{\circ}\right)$. Além disso, os valores obtidos da $P_{\mathrm{d}}$ em relação à rampa superior foram baixos. Porém, comparando os resultados dos dois tipos de dispositivo (duas rampas e rampa única), o dispositivo de rampa única mostrou-se a melhor opção, quando submetido às condições de contorno impostas, pois apresentou para $P_{\mathrm{d}}$ um valor 11,25 vezes maior ao encontrado para o dispositivo de galgamento com rampa dupla. Concluiu-se que a rampa superior funcionou como uma restrição, impedindo uma maior entrada de água no reservatório, fazendo com que grande parte a água voltasse ao mar. Desta forma, estudos considerando outras configurações para o dispositivo de rampa dupla são necessários para um melhor julgamento da determinação da Potência Máxima Disponível.

\section{AGRADECIMENTOS}

O autor JCM agradece a CAPES pela bolsa de estudos. Os autores LAOR, LAI e EDS agradecem ao CNPQ pelo suporte financeiro.

\section{REFERÊNCIAS BIBLIOGRÁFICAS}

1. Cruz JMBP, Sarmento ANA. Energia das Ondas: Introdução aos Aspectos tecnológicos, Econômicos e Ambientais. Instituto Superior Técnico - Wave Energy Centre. Amadora, Lisboa; 2004. 61 p.

2. Fleming FP. Avaliação Do Potencial De Energias Oceânicas No Brasil. [dissertação]. Rio Grande (RS): Universidade Federal do Rio Grande, 2012.

3. Margheritini L, Vicinanza D, Frigaard P. SSG wave energy converter: Design, reliability and hydraulic performance of an innovative overtopping device. Renew Energy. 2009;34(5):80-1371, doi:10.1016/j.renene.2008.09.009

4. Vicinanza D, Contestabile P, Quvang Harck Nørgaard J, Lykke Andersen T. Innovative rubble mound breakwaters for overtopping wave energy conversion. Coast Eng. 2014;88:70-154, doi:10.1016/j.coastaleng.2014.02.004

5. Liu Z, Han Z, Shi H, Yang W. Experimental study on multi-level overtopping wave energy convertor under regular wave conditions. Int J Nav Archit Ocean Eng. 2017;1-9, doi:10.1016/j.ijnaoe.2017.10.004

6. Contestabile P, Iuppa C, Di Lauro E, Cavallaro L, Andersen TL, Vicinanza D. Wave loadings acting on innovative rubble mound breakwater for overtopping wave energy conversion. Coast Eng. 2017;122(January):60-74, doi:10.1016/j.coastaleng.2017.02.001

7. Margheritini L, Hansen AM, Frigaard P. A method for EIA scoping of wave energy converters-based on classification of the used technology. Environ Impact Assess Rev. 2012;32(1):33-44, doi:10.1016/j.eiar.2011.02.003

8. Nørgaard JH, Andersen TL. Investigation of Wave Transmission from a Floating Wave Dragon Wave Energy Converter. International Society of Offshore \& Polar Engineers; 2012: p.16-509.

9. Barbosa DVE, Souza JA, Isoldi LA, Martins JC. Numerical analyses of openfoam's overtopping device solution. Reterm. 2017;16(1):96-103, doi: 10.5380/reterm.v16i1.6219

10. Jungrungruengtaworn S, Hyun BS. Influence of slot width on the performance of multi-stage overtopping wave energy converters. Int J Nav Archit Ocean Eng. 2017;9(6):668-76, doi:10.1016/j.ijnaoe.2017.02.005 
11. Goulart MM, Martins JC, Junior ICA, das Neves Gomes M, Souza JA, Rocha LAO. Constructal design of an onshore overtopping device in real scale for two different depths. Mar Syst Ocean Technol. 2015;10(2):120-9, doi:10.1007/s40868-015-0010-7

12. Martins JC, Goulart MM, Souza JA, Isoldi LA, Santos ED, Gomes MN, Rocha LAO. Constructal design of an onshore overtopping device in real scale for different ramp construction areas. Proceedings of the 23rd ABCM International Congress of Mechanical Engineering; 2015; Rio de Janeiro.

13. Machado BN, Dos Santos ED, Isoldi LA, Gomes MDN, Rocha LAO. Análise numérica da geometria da rampa de um dispositivo de galgamento onshore em escala real aplicando o design construtal. Rev Bras Energias Renov. 2017;6(3).

14. Martins JC, Dos Santos ED, Isoldi LA, Gomes MDN, Rocha LAO. Geometric evalution of the ramp of an overtopping device by means of constructal design and a wave. Proceedings of the XXXVIII Iberian Latin-American Congress on Computacional Methods in Engineering. Florianópolis - SC; 2017.

15. Martins JC, Goulart MM, Gomes M das N, Souza JA, Rocha LAO, Isoldi LA, et al. Geometric evaluation of the main operational principle of an overtopping wave energy converter by means of Constructal Design. Renew Energy. 2018;118:727-41, doi:10.1016/j.renene.2017.11.061

16. Bejan A. Shape and structure, from engineering to nature. Cambridge University Press; 2000.

17. Bejan A, Lorente S. Design with Constructal Theory. John Wiley \& Sons; 2008.

18. Ansys. Ansys Fluent: Theory Guide; 2015.

19. Almeida LESB, Rosauro NML, Toldo Jr. EE. Análise Preliminar das Marés na Barra do Rio Tramandaí. Anais do XII Simpósio Brasileiro de Recursos Hídricos. Vitória, ES. Brasil; 1997. p.560566.

20. Lisboa R da C, Teixeira PR de F, Didier E. Simulação da propagação de ondas regulares e irregulares em um canal bidimensional com praia numérica. Anais do VII Seminário e Workshop em Engenharia Oceânica. Rio Grande, RS, Brasil; 2016.

21. Hirt CW, Nichols BD. Volume of fluid (VOF) method for the dynamics of free boundaries. J Comput Phys. 1981;39(1):201-25.

22. Schlichting H, Gersten K. Boundary-Layer Theory. Berlin, Heidelberg: Springer Berlin Heidelberg; 2017; doi:10.1007/978-3-662-52919-5.

23. Chakrabarti SK. Handbook of Offshore Engineering. Amsterdam, Netherlands: Elsevier; 2005.

24. Dean RG, Dalrymple RA. Water Wave Mechanics for Engineers and Scientists. Toh Tuck, Singapore: World scientific; 1991.

25. McCormick ME. Ocean engineering wave mechanics. Nova Jersey, EUA: Wiley; 1973.

26. Patankar S V. Numerical heat transfer and fluid flow. Hemisphere Pub. Corp.; 1980. 197 p.

27. Versteeg HK, Malalasekera W. An introduction to computational fluid dynamics: the finite volume method. Pearson Education Ltd; 2007.

28. Mavriplis DJ. Unstructured Mesh Generation and Adaptivity. In.: 26th Computational Fluid Dynamics Lecture Series. Program of the von Karman Institute for Fluid Dynamics. Rhode-Saint-Genese, Belgium; 1995.

29. Gomes M das N, Isoldi LA, Santos ED dos, Rocha LAO. Análise de malhas para geração numérica de ondas em tanques. Anais do VII Congresso Nacional de Engenharia Mecânica - CONEM 2012. São Luís, MA, Brasil; 2012.

30. Kreyszig E, Kreyszig H, Norminton EJ. Advanced engineering mathematics. Jefferson City, Missouri: Wiley; 2011.

31. Gomes M das N, Costa CA de O, Deus MJ de, Ricardo RLP, Isoldi LA, Santos ED. Análise computacional e geométrica com design construtal de um dispositivo conversor de energia das ondas do mar em energia elétrica do tipo coluna de água oscilante submetido a um espectro de ondas. Proceedings of XXXVII Iberian Latin American Congress on Computational Methods in Engineering CILAMCE 2016. Brasília - Brasil; 2016. 